\title{
Preying on beauty? The complex social dynamics of overtourism
}

\author{
Angelo Antoci ${ }^{1}$ - Paolo Russu ${ }^{1} \cdot$ Pier Luigi Sacco ${ }^{2,3,4}$ • \\ Giorgio Tavano Blessi ${ }^{2,5}$
}

Received: 9 May 2020 / Accepted: 7 December 2020 / Published online: 23 February 2021

(c) The Author(s), under exclusive licence to Springer-Verlag GmbH, DE part of Springer Nature 2021

\begin{abstract}
Overtourism is an increasingly relevant problem for tourist destinations, and some cities are starting to take extreme measures to counter it. In this paper, we introduce a simple mathematical model that analyzes the dynamics of the populations of residents and tourists when there is a competition for the access to local services and resources, since the needs of the two populations are partly mutually incompatible. We study under what conditions a stable equilibrium where residents and tourists coexist is reached, and what are the conditions for tourists to take over the city and to expel residents, among others. Even small changes in key parameters may bring about very different outcomes. Policymakers should be aware that a sound knowledge of the structural properties of the dynamics is important when taking measures, whose effect could otherwise be different than expected and even counterproductive.
\end{abstract}

Keywords Overtourism · Tourist destinations · Congestion · Tourists versus residents $\cdot$ Nonlinear dynamics

JEL Classification C02 $\cdot$ C63 $\cdot$ Z38

$凶$ Pier Luigi Sacco

pierluigi.sacco@iulm.it

1 Department of Economics and Business (DiSea), University of Sassari, Sassari, Italy

2 Department of Humanities, IULM University Milan, Via Carlo Bo, 1, 20143 Milan, Italy

$3 \mathrm{CHuB}, \mathrm{FBK}$ Foundation, Trento, Italy

4 Berkman-Klein Center for Internet and Society, Harvard University, and metaLAB (at) Harvard, Cambridge, MA, USA

5 Faculty of Education, Free University of Bozen, Bolzano, Italy 


\section{Introduction}

The rapid increase of global mobility that has characterized the mature phase of the globalization process in the past couple of decades has also, as a consequence, led to the escalation of 'overtourism' issues in many global tourism destinations, and most notably in major art and heritage cities. Despite massive flows of tourists clearly benefit the local economy, they also pose a major threat to both the livability and, in some cases, even the sustainability of cities that are literally consumed by a level of human occupancy they weren't designed or intended to host. In Barcelona, where the number of overnight stays escalated from 1.7 millions in 1990 to more than 8 millions in 16 years, overtourism is one of the key causes of an environmental pollution emergency (Ledsom 2019). In addition to the most renowned tourist locations, the geography of overtourism is also rapidly expanding due to the global visibility acquired by some cities for having been the shooting location of successful TV series, as in the case of Dubrovnik for Game of Thrones (Wiley 2019). However, an increasing number of critical voices are questioning this trend, locally as well as internationally (Economist, 2018). For residents, overtourism may have dramatic consequences. Housing for permanent residential use becomes increasingly scarce and expensive. Services catering to the needs of locals become rarer, more difficult to reach, and again more expensive. The constant noise and the overcrowding of streets and local transport can be a source of considerable stress for working people, families with small children and the elderly. In cities like Venice, the number of bed-and-breakfasts and flats for short-term tourist occupancy has nearly doubled in the space of just one year (Tantucci 2018). As a consequence, residents are evicted by landlords who find way more profitable to rent to tourists. In Florence, for instance, between October 1, 2017, and June 30, 2018, as many as 478 residents who couldn't keep up with the rising rents had to leave their homes, including lifetime ones: 209 living in the historical center, 71 in the Unesco area and 198 in other areas of the city (Conte 2018). More generally, the so-called airification (Picascia et al. 2019) has been identified as a disruptive force that is literally 'hollowing out' cities (Hinsliff 2018). Such a state of things does not come as a complete surprise to the tourism studies literature. Although early warnings were appropriately sent, as in the seminal paper by van den Borg et al. (1996), they have not succeeded in convincing local policy makers to devise appropriate countervailing strategies and to take action.

Now that the negative effects of the phenomenon are becoming indisputable, however, some cities are starting to react aggressively. Amsterdam has banned the concession of new licenses to business within the historical city core that offer goods and services targeting tourist demand (O'Sullivan 2017), as a way to curb the 'Disneyfication' of the city (Boztas 2017). Bruges has strictly limited the maximum number of cruise ships that may be hosted at its port's docks on a daily basis and has limited its own tourism-related advertising in major nearby cities (Marcus 2019). Venice has implemented a very severe set of restrictions to many different kinds of tourist misbehavior, sanctioned with heavy fines (Spinks 2018). Ten major European heritage cities such as Amsterdam, Barcelona, Berlin, Bordeaux, Brussels, Krakow, Munich, Paris, Valencia and Vienna have jointly signed a letter to the new EU Commission asking for severe limitations to the further expansion of Airbnb and other holiday rental websites 
(Henley 2019). However, it is not easy to go against such a powerful trend, despite that the current COVID-19-related crisis that has caused a temporary collapse of the tourism industry worldwide will probably provide overcrowded tourist cities with an unexpected opportunity to prevent the eventual return to the 'old normal' once the pandemic is over (Higgins-Desbiolles 2020). The vested interests that rely upon the extractive logic of the mass tourism economy are a major local consensus pool and exert powerful political pressure (Benner 2019). On the other hand, the needs of tourists and residents significantly differ, and this is likely to spark conflict between different local stakeholders, depending on the extent to which they benefit from tourism (Concu and Atzeni 2012). Whether or not a city eventually gets colonized by the tourism economy or manages to find a reasonable compromise can therefore be the result of a very complex interplay of factors. It is therefore of particular importance to study under what conditions such interplay leads to different long-term scenarios, thus enabling public decision makers to better understand not only the nature of the problem in order to imagine and test possible solutions, but also the critical conditions that regulate the emergence of possible outcomes. Merely proposing 'plausible' or 'just' solutions is not enough. We also need to assess whether such solutions would work, and under what circumstances, once they are actually implemented. In principle, solutions that are more desirable in abstract terms need not be the ones that work best. As cities are very complex dynamical systems, the pursuit of the public interest, which in this case identifies to a significant extent with that of city residents, whose 'right to the city' (Lefebvre 2010) should be the object of special consideration and protection, needs to be supported by evidence-based policies building upon a sound understanding of the underlying economic and social dynamics.

The aim of this paper is that of studying a simple dynamic model that analyzes the effects of the tension between residents and tourists in the social usage of city resources. We focus on the interplay of the essential factors behind such tension: the substitution between resident-oriented and tourist-oriented facilities and shops, the congestion of city space from overtourism, but also the experience value of cities as related to the effective presence of residents as a source of authenticity. Given that the escalating tourist flows are literally preying on the city's resources from the residents' viewpoint, it is natural to think of modeling such dynamics with the predatorprey framework in mind. We introduce an expanded variant of the predator-prey dynamics, which yields more complex dynamic behavior than the original one, and allows a better analytical treatment of the main factors at play. The model's structure is easily interpretable, but the corresponding dynamics are not obvious. In particular, we show that the actual dynamic trajectories of the system may be very different for relatively small changes in the key parameters. This implies that even relatively small differences in local conditions and in policy actions may cause divergent outcomes, with substantial differences in terms of their social desirability. Our results should be read as a cautionary tale against delayed or unsystematic action in curbing the social costs from overtourism: intervening too little or too late, or not focusing on the truly critical parameters might lead to disappointing results.

The remainder of the paper is organized as follows. Section 2 offers a brief review of the main issues discussed in the overtourism-related literature. Section 3 presents 
the model. Section 4 contains the main results. Section 5 discusses the results and concludes. A technical Appendix closes the paper.

\section{Literature review}

One vastly debated issue that clearly relates to overtourism is that of residents' attitudes toward tourists. There is a rich literature that explores this topic, but most of it has dealt with minor or even marginal tourist destinations rather than with overcrowded tourist attractors. Lin et al. (2017) focus upon the process of value co-creation through social interaction between tourists and residents in a Chinese sample and find that the positive economic benefits from tourism may also positively affect the life satisfaction of residents. Mathew and Sreejesh (2017), working on a sample of three Indian tourism destinations, highlight the relationship between responsible tourism and perceptions of sustainability of the tourist destination in promoting the perceived quality of life of residents. On the other hand, Boley et al. (2017) show that although destinations that place more emphasis on sustainability tend also to be the more sustainable, perceptions of actual sustainability by residents tend to be low. Rasoolimanesh et al. (2017) show that, for two UNESCO Heritage sites in Malaysia, one of which located in an urban context and the other in a rural one, there are nuanced differences between the urban and the rural site in terms of the impact of residents' perceptions on the support for tourism development or lack thereof, but also substantial homogeneities. Therefore, when tourism is still in a developing phase, the evidence of the benefits from tourism development can be a main driver of support from residents, and this effect may even cut across major territorial divides such as the urban/rural one. As shown by Stylidis et al. (2014) and Wang and Chen (2015), a central mediating role in residents' perceptions of the impacts of tourism is perceived place image-a dimension that is, tellingly, significantly compromised in destinations affected by overtourism, but can be improved by an increased tourists presence in developing destinations. It is no surprise that literature reviews of this research field lament the excessive narrowness of focus of most research, as well as its reliance on specific quantitative techniques that are good at highlighting specific effects but often fail to deliver the big picture (Sharpley 2014). For instance, Almeida Garcia et al. (2015) argue that the current literature on residents' perception of tourism significantly underplays the role of key historical, cultural and social factors in shaping a specific destination and its response to tourism. Moreover, the nature of the 'ecological' interactions between the residents and tourists populations may make a big difference and has an intrinsically dynamic nature (VargasSanchez et al. 2011). And if this is true in general, it is even truer for overcrowded destinations, and the possible scenarios may be very different from one another. For instance, touristic congestion may be the result of a sudden boom or of a gradual, steady increase; the pervasive presence of tourists in the urban space may have become a deeply ingrained feature of the local culture, or be an outcome of recent tourism development strategies; the availability of space and the impact of building density may be not particularly problematic for urban livability or rather extremely critical and exacerbated by tourism flows, and so on, just to limit ourselves to a few obvious examples. Segota et al. (2017) show for instance that the informedness and involvement 
of residents in the local management of tourism-related issues significantly impacts their perceptions in the expected direction (the more involved and informed, the more positive). A rare example of a study on the acceptability of crowding perceptions by residents in a global tourist destination such as Bruges, carried out by Neuts and Nijkamp (2012) , moreover, shows that the actual negative perception of crowding varies widely across residents depending on individual characteristics and is not found in the majority of the sample. However, the situation might have changed now, in the light of further recent accelerations of tourism flows in many heritage cities, as possibly signaled by Bruges' current de-advertising on the tourism market. Despite this, overtourism and its policy implications are still relatively poorly covered in the literature, with the consequent risk of failing to fully appreciate the complex social conflict issues that can emerge and deflagrate in the absence of proper policy strategies and management at the city level.

The key critical aspect, which is amplified by overtourism but already apparent in developing destinations even in the case of positive residents' perceptions, is the impact of tourism on local culture and behaviors, whose effects can only be appreciated in full in the medium-long run. Of course, culture and behaviors are inevitably bound to change anyway, independently of tourism. But the changes induced by tourism might eventually clash with the developmental priorities and goals of local communities (Simpson 2009. There is a need to strike a balance between the benefits of tourism as a local developmental driver and potentially negative effects, e.g., in terms of longlasting impacts on cultural identity and authenticity (Lacy and Douglass 2002; Cole 2007; Zhu 2012), on socioeconomic inequalities (Lee 2009; Alam and Paramati 2016), on community empowerment (Cole 2006; Aref and Redzuan 2009; Chen et al. 2017), and so on. Especially critical is the evaluation of residents' perceptions in developing countries affected by substantial socioeconomic issues (Truong et al. 2014). Analyses that rely on an exclusively tourism-centric perspective are likely to overlook the most critical dimensions (Easterling 2004). Ribeiro et al. (2017)'s analysis of the development of pro-tourism behaviors of Cape Verde Islands residents is an example in this regard. Nunkoo and Gursoy (2012) instead consider, in the case of Mauritius, the role of local identity in the orientation of residents' support for tourism, but interestingly point out how even the emergence of a supportive orientation need not reflect into a significant shift in attitudes, thus underlying the complex functioning of community identity as a regulator of cultural and social change. On the other hand, tourism itself is constantly raising the bar as to the level and depth of interaction with local social life and customs that tourists expect to reach as a quintessential aspect of their experience, to the extent of becoming co-creators of the experience itself. Prebensen and Xie (2017) show, for example, that the level of tourists' participation under the form of mastering and co-creation in experience tourism significantly enhances their value perception. Paulaskaite et al. (2017) highlight how tourists increasingly expect to spend their time at the destination 'living like the locals,' therefore transforming local identity itself into a commodity that can be purchased at will.

Such issues are relevant for all kinds of tourist destinations, but they are especially problematic in overcrowded ones. Overtourism shifts the focus of residents' perceptions on critical aspects such as the pressure of tourism flows on the local system (Muler Gonzalez et al. 2018), the threats to ecological sustainability (Cheer et al. 
2019) and the role of media, and social media in particular, in causing tourist congestion peaks on an almost instantaneous basis (Jang and Park 2020). In other words, the aspect of overtourism that is seen as the most socially alarming is its capacity to put under stress at an unprecedented scale and pace the homeostatic mechanisms of local systems on many different levels: economic, social, cultural, logistical, and so on. Overtourism magnifies many of the most critical features of tourism to an extent that strains local governance and regulatory capacity; however, its effects may be more critical on certain dimensions rather than others (Carvalho et al. 2020). When such impact is perceived as disruptive by local communities, social protest ensues (Alexis 2017; Pinkster and Boterman 2017; Seraphin et al. 2018). Once a perceived saturation level is reached, a vicious circle can take over as residents classify as threatening by default any tourism event that causes local congestion, irrespectively of its quality, importance and expected long-term benefit for the city (Lemmi et al. 2018). This kind of vicious circle may mutually reinforce with others, e.g., the one causing the erosion of local services quality in overcrowded tourism destinations (Caserta and Russo 2002). Such social dynamics are difficult to manage at all levels, and even large digital tourism platforms may find it hard to function well (e.g., in rewarding quality in their rankings of local businesses) when the effects of digital influencing upon spatial patterns of tourism congestion spark social controversy (Ganzaroli et al. 2017). Such new, system-wide challenges may be effectively tackled only through tailored, sophisticated forms of local cooperation between key stakeholders (Kuscer and Mihalic 2019) and of smart governance (Agyeiwaah 2019).

\section{The model}

The literature briefly discussed in the previous section shows how the problem of overtourism, in the more general context of the residents' perceptions of the social and economic impacts of tourism, is generally focused on the analysis of specific case studies and on the measurement of perceptions and attitudes by means of suitable psychometric tools. In this paper, we take a different route as a contribution to a comprehensive approach to the smart governance of overtourism dynamics: that of characterizing such dynamics in terms of an explicit mathematical model. The ambition of the model is not to provide a detailed, realistic representation of overtourism in all of its multifaceted dimensions, but to examine what are the basic conditions that may favor, or prevent, its onset, paying special attention to a basic phenomenon: the competition between resident-oriented and tourist-oriented services for the limited spatial and material resources of the city. As we have seen from the literature review, a detailed modeling of such dynamics would involve many different variables-place identity, social perceptions, local culture, historical trajectories and many more-and this would easily make an explicit dynamic analysis intractable due to the number of potential variables implied. However, simplifying the model to its essentials has the advantage of providing some insight that may help focus upon the possible dynamic regimes that may prevail, providing policymakers with some important indications for policy design. 
We choose as our conceptual benchmark the classical Lotka-Volterra predator-prey model, which has been the object of countless applications in a variety of different fields, and of mathematical generalizations of all kinds due to its optimal combination of simplicity of structure and richness of dynamic behaviors. In our case, the predatorprey logic is somewhat ingrained into the nature of the problem we want to analyze, as one thinks of overtourism as the process through which tourism flows literally 'capture' the local system, reshaping it according to their necessities. On the other hand, even a basic description of the overtourism problem urges us to depart from the basic formulation of the predator-prey model to better take account of some essential specificities. In particular, the model we propose has the following structure:

$$
\begin{aligned}
& \dot{x}=r+a x-b(y-\bar{y}) x+c(x-\bar{x}) \\
& \dot{y}=s+d x y+e(y-\bar{y}) x-f y
\end{aligned}
$$

where $x$ is the level of the resident population and $y$ is the level of the tourist population. All parameters are positive. Let us now see in some detail the rationale behind the equations. The basic premise of the model is that there is an implicit competition between residents and tourists for the availability of services and resources that respond to their specific, and partly mutually incompatible, needs. In particular, there are two threshold values of $x$ and $y, \bar{x}$ and $\bar{y}$, respectively, beyond which the local level of residents (tourists) is large enough to warrant a satisfactory provision of resident(tourist-) specific services and resources. We call such thresholds the relevance thresholds. When one population crosses its relevance threshold, the local economy becomes increasingly respondent to that population's needs, and this positively influences the dynamics of such population. These two effects are captured, respectively, by the two terms $x-\bar{x}$ and $y-\bar{y}$. So, the level of the resident population is positively depending on whether the residents are above their relevance threshold, and negatively depending on whether the tourists are above their relevance threshold. In this latter case, however, the size of the effect is scaled by the level $x$ of the resident population: the larger the pool of residents, the more an above-threshold level of tourists makes competition for scarce space and resources more sustained, increasing the negative impact of tourists on the resident population. Parameters $b$ and $c$ measure the relative size of the two effects. Moreover, the dynamics of the resident population also linearly depends (according to the parameter $a$ ) on the actual level of the resident population, as the choice to live in a city is characterized by some amount of inertia, due to a variety of factors such as relocation costs, habit, cultural and affective reasons, job-related reasons, and so on. As to the tourist population, it positively benefits from the crossing of its own relevance threshold as already anticipated, and the effect is measured by the parameter $e$. Moreover, its dynamics are negatively influenced by tourism congestion, an effect whose size is measured by the parameter $f$. Finally, the tourist population's persistence in the destination also depends, and in a positive way, on the level of residents, as measured by the parameter $d$. Insofar as the resident population is small, the city basically turns into a 'theme park' devoid of any specific authenticity and vitality, to become a mere entertainment district that maximizes tourism-related profit. This 
effect, as already hinted at in the discussion of the previous section, therefore captures the 'experience economy' dimension, as tourists do not simply ask for entertainment, but also value opportunities of meaningful interaction with locals. Finally, parameters $r$ and $s$ measure the exogenous components of the rates of growth of the resident and tourist populations, respectively.

The system of equations above can be conveniently rewritten as follows:

$$
\begin{aligned}
& \dot{x}=r-c \bar{x}+(a+b \bar{y}+c) x-b x y \\
& \dot{y}=s-e \bar{y} x+(e+d) x y-f y
\end{aligned}
$$

In our analysis, we will refer to (1)-(2) as the default formulation of the model.

\section{Existence and stability of the stationary states}

To analyze the dynamic behavior of the model, we start by posing:

$$
A:=\frac{a+b \bar{y}+c}{b}, \quad B:=\frac{c \bar{x}-r}{a+b \bar{y}+c}, \quad C:=\frac{e \bar{y}}{e+d}, \quad D:=\frac{s}{e \bar{y}}, \quad E:=\frac{f}{d+e}
$$

The complete taxonomy of possible dynamic regimes is illustrated in the following proposition. We will see how even a relatively simple model like the present one can generate a rich array of dynamic behaviors depending on the prevalence of certain constellations of conditions rather than others.

Proposition 1 Under the assumption that all parameters of the system (1)-(2) are strictly positive, the following dynamic regimes can be observed:

(1) If $B>0$ (i.e., $\bar{x}>\frac{r}{c}$ ), then at most two stationary states exist. In particular,

(1.a) if either $D<B<E$ or $E<D<B$ holds, a unique repelling stationary state $P$ exists (Fig. $7 b, c)$ in the Appendix);

(1.b) if $B<\min \{D, E\}$, two stationary states $P_{1}=\left(x_{1}^{*}, y_{1}^{*}\right)$ and $P_{2}\left(x_{2}^{*}, y_{2}^{*}\right)$, with $x_{1}^{*}<x_{2}^{*}$ and $y_{1}^{*}<y_{2}^{*}$, may exist, where $P_{1}$ is always a saddle point, whereas:

(1.b.1) if $D<E$, then $P_{2}$ is a repeller (Fig. 7a) and (7e) in the Appendix);

(1.b.2) if $D>E$, then $P_{2}$ is either a repeller or an attractor (Fig. $7 d$ in the Appendix).

(2) If $B<0$ (i.e., $\bar{x}<\frac{r}{c}$ ) a unique stationary state exists; if $D<E$, it is a either a repeller or an attractor (Fig. $7 f$ in the Appendix) while, if $D>E$, it is an attractor (Fig. $7 g$ in the Appendix).

Proof See Appendix

To explain the meaning of Proposition 1, let us start by understanding better the interpretation of the new composite parameters $A, B, C, D$ and $E$. The parameter 
$A$ measures the relative size of the parameters that positively regulate the growth of the resident population vs. the parameter $b$ that negatively affects it. In particular, the growth of the size $x$ of the resident population depends positively on the parameter $a$ (measuring the persistence effect), on the parameter $c$ (representing the reactivity to the difference between $x$ and the threshold $\bar{x}$ and on the parameter $\bar{y}$ (the threshold for the tourist population). We can therefore intuitively interpret $A$ as a measure of residents' resilience. As for $B$, it positively depends on the relevance threshold of the resident population (measured by $c \bar{x}$ ): the larger it is with respect to $r$, and with respect to the parameters that positively influence residents' resilience, the larger $B$. $B$ can therefore be intuitively interpreted as a measure of residents' susceptibility: the higher $B$, the more demanding for the residents' community to fulfil the conditions for the prevalence of a resident-oriented local economy. Likewise, $C$ can be interpreted as a measure of tourists' susceptibility, as $C$ is larger the higher the threshold of relevance for tourists $e \bar{y}$, and the smaller the combined strength of the experience value parameter $d$ from visiting the city plus the impact $e$ of crossing the relevance threshold on the availability of tourist-oriented services and resources. $D$ can be seen as the city's intrinsic attraction value for tourists, as it equals $s$ (the exogenous growth rate of tourists) scaled by the relevance threshold for tourists. Finally, $E$ measures the tourists' relative congestion effect expressed by the congestion parameter $f$ scaled by the combined strength of the experience value and resource and service availability effects for tourists.

At this point, we are ready to illustrate the findings in Proposition 1 . The results are organized around the sign of $B$, that is, whether or not the residents' susceptibility problem occurs, which implies a relatively high relevance threshold for the residents-oriented local economy to kick off. In the case of a positive level of residents' susceptibility, that is, $B>0$, we have at most two stationary states that can be potential equilibria for the dynamics. A first sub-regime relies on two possible conditions at which, of the two possible stationary states, only one exists and is repulsive, that is, the dynamics never settle down to a given state. The two conditions are $D<B<E$ and $E<D<B$. In the first case, we have a condition where the congestion effects is particularly high with respect to residents' susceptibility and to the intrinsic attraction value for tourists. This is, for example, the case of a relatively small city where, despite the comparatively modest attraction value, congestion is a problem and tourists can crowd out residents relatively easily. In the second case, we have to the contrary a situation where congestion is relatively unimportant and residents' susceptibility is comparatively high in presence of a relatively substantial attraction value. This is for instance a scenario that could describe a relatively large city with high carrying capacity and cultural/amenity value, where there is real competition for local resources and services between residents and tourists. These two conditions may therefore span very different cases.

A second sub-regime contemplates again the existence of two possible stationary points, one of which is always a saddle, that is, a state where a unique converging trajectory exists and all the other ones diverge. The key condition for the second sub-regime is that $B$ be smaller than both $D$ and $E$. Given that $B$ is constrained to be positive, the condition requires that both the congestion effect and the intrinsic attraction value are relatively high. An example here is that of an established tourism 
destination with severe congestion problems where however the issue of resource accessibility for residents is relatively less binding, possibly due to a large, diversified local economy that can accommodate local demand. An extra condition regulates the dynamic properties of the second possible steady state, according to the relative size of the two potentially dominating effects. If the dominant effect is congestion, the second stationary state is locally unstable. If instead the dominant effect is the intrinsic attraction value, the second stationary state may either be locally unstable or locally stable, that is, may attract all local trajectories and emerge as a stable state.

When residents' susceptibility is not a major concern (i.e., $B<0$ ), the dynamic regime is much simpler. In this case, the stationary state is always unique. Moreover, if congestion prevails upon intrinsic attraction, this state may be either attracting or repelling (locally stable vs. unstable). If the opposite is true and intrinsic attraction prevails, the stationary state is always attractive. An example of this latter condition is a world-renowned tourist destination, with a large carrying capacity that can manage congestion, and where the competition between residents and tourists for local services and resources is not binding.

Proposition 1 tells us, among other things, that the dynamics we are studying is not in many cases conducive to a stable equilibrium state and is rather characterized by more complex long-run behaviors. The stability properties of the stationary states do not give us enough information to understand what such dynamic behaviors will look like, as they only provide insight about what happens close to them. However, the structure of stationary states is an important piece of information, and in particular, it is interesting to ask how the number and stability properties of the stationary states vary depending on the levels of specific couples of parameters such as the relevance thresholds for residents and tourists, given our focus on overtourism and its possible impacts. In all the analysis that follows, the choice of parameter values for the simulations has been made in order to select cases that enable us to illustrate clearly and in a compact way the dynamic properties of the model.

Figure 1 illustrates the bifurcation diagrams obtained by varying the relevance threshold $\bar{y}$. Panels (a) and (b) show how the coordinates $x$ and $y$ (on the horizontal axis) of stationary states vary in response to variations in $\bar{y}$ (on the vertical axis). The LP point separates the interval of $\bar{y}$ values where no stationary state exists, from that in which two stationary states exist. The point $\mathrm{H}$ indicates the Hopf bifurcation value of $\bar{y}$. Dashed, continuous and dotted lines represent saddle points, attractive and repulsive stationary states, respectively. The conditions under which a Hopf bifurcation occurs by varying the parameter $\bar{y}$, computed according to the criterion proposed by Liu (1994), are given in the Appendix. In Panel (c) of Fig. 1, we show how, through the Hopf bifurcation, a family of limit cycles emerges. Notice that an increase in the parameter value $\bar{y}$ leads to an increase in the magnitude of the limit cycles.

In Fig. 2, we show, for a specific set of parameter values, the bifurcation diagram that illustrates the existence and stability of the stationary states as the two relevance thresholds vary. Figure 2 a provides the full diagram, whereas Fig. 2 b presents an enlargement of the rectangle area where the most fine-grained structure is found. As we can see, the bifurcation diagram contains here all seven possible scenarios for the stationary states, where, in Fig. 2, the apexes $(S, A),(S, R), A, R$ denote, respectively: regions where two stationary states exist, of which one is a saddle $(\mathrm{S})$ and another an attractor (A); 


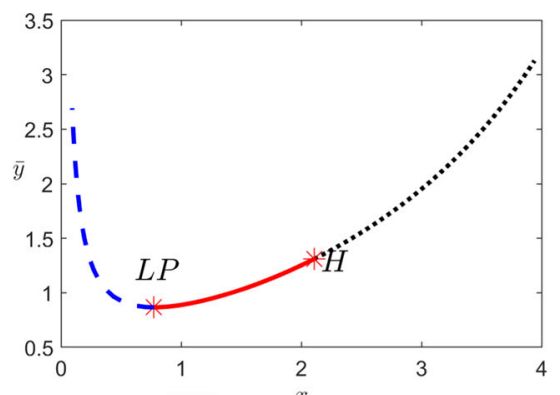

(a)

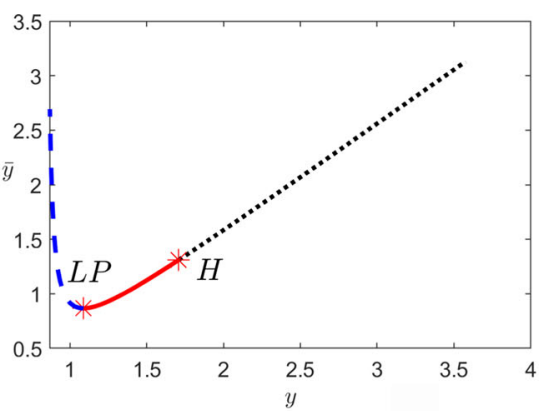

(b)

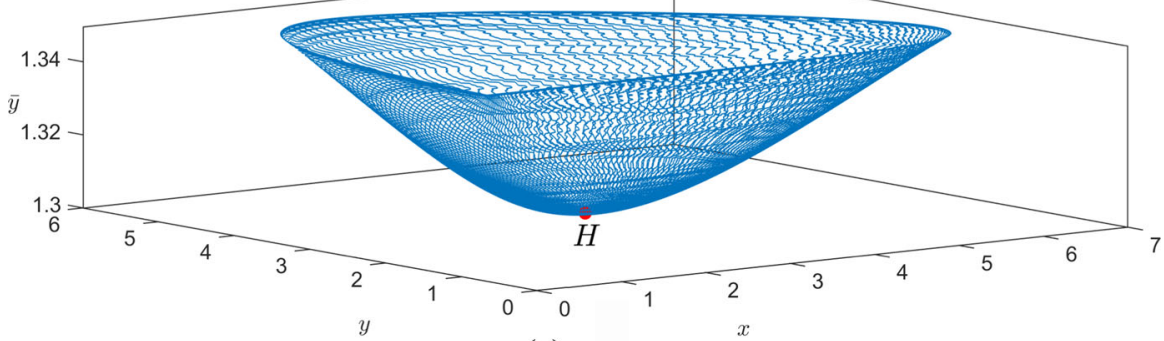

(c)

Fig. 1 Bifurcation diagrams obtained by varying the relevance threshold $\bar{y}$. Panels $\mathbf{a}$, b show how the coordinates $\mathbf{x}$ and $\mathbf{y}$ (on the horizontal axes) of stationary states vary in response to variations in $\bar{y}$ (on the vertical axis). Panel c shows the family of limit cycles that emerges through the supercritical Hopf bifurcation. Parameter values: $a=2, b=7, c=1.5, d=2.5, e=5, f=16.5, r=3, s=15, \bar{x}=3$

regions where two stationary states exist and are in particular a saddle and a repeller (R); regions where one stationary state exists and is an attractor; and regions where one stationary state exists and is a repeller. The $\mathrm{H}$ curve is the Hopf bifurcation curve, whereas the LP curve is the one that separates the region without stationary states from the region where at least one stationary state exists. The Hopf bifurcation curve $\mathrm{H}$ separates the regions where an attractive stationary state is found (to the left of the curve) from those where a cycle emerges, as shown in more detail in Fig. 2b. In the simulations below, we find that the attractive cycle is stable and the corresponding stationary state consequently becomes unstable. Figure 2c, instead, reports the bifurcation diagram in the $(c, e)$ space, where we study how the structure of stationary states varies with the parameters that measure the strength of resource provision when residents (respectively, tourists) cross their relevance threshold. Again, the bifurcation curve $\mathrm{H}$ and the LP curve delimit the areas where one of the stationary states (or the only one, if unique) changes its local behavior from repulsive to attractive, and where stationary states exist vs. fail to exist.

From these figures we see how, in the case of the bifurcation diagram for the relevance thresholds, there is a vast region where stationary states do not exist for most values of the relevance threshold for residents if the relevance threshold for tourists is small enough. That is, when tourists are substantially favored in their capacity to access local resources with respect to residents, the dynamics fails to settle on a stationary state. However, when the relevance threshold for residents is very low, even 


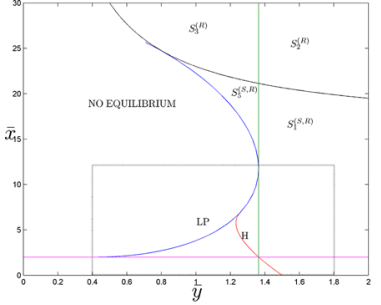

(a)

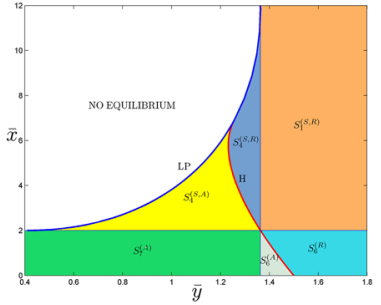

(b)

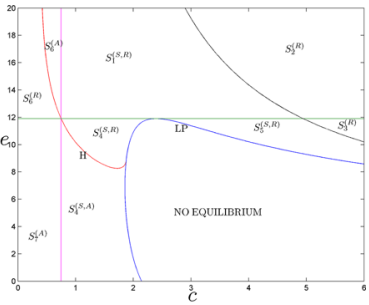

(c)

Fig. 2 Existence and stability of the stationary states in (a) $(\bar{y}, \bar{x})$-plane, (c) $(c, e)$-plane; (b) shows an enlargement of the rectangle indicated in (a). Parameters are: $a=2, b=7, c=1.5, d=2.5, e=5$, $f=16.5, r=3, s=15$

for relatively high levels of the relevance threshold for tourists a stable stationary state emerges. That is, when residents succeed in getting access to the local resources, the system has a chance to stabilize itself. But when the relevance threshold for tourists or even both thresholds become very high so that it is difficult for both populations to gain easy accessibility to local resources, there is no chance that the system may settle down to a stable equilibrium.

In the case of the bifurcation diagram in the $(c, e)$ space, the pattern is more complicated, and the existence of stable stationary states here relies on more specific combinations of the two parameters. In general, when $c$ is very high, that is, when the access to resources beyond the relevance threshold has a big positive impact on the population of residents, no equilibrium exists, whereas for smaller values of $c$ a stable stationary state can emerge. Again, when both parameters are large, no stable stationary state can be found. Remember that these bifurcation diagrams are drawn for a given choice of numerical values of all the other parameters, and that they change as any one of the other parameters varies.

To get a better understanding of what the actual trajectories of the system look like, we report a few examples of phase diagrams for a specific choice of parameter values in Fig. 3. In particular, we keep the values of all the other parameters but the relevance thresholds as in Fig. 2, and we set a specific value for $\bar{x}=4$, letting $\bar{y}$ vary. The four cases correspond, respectively, to points from the white, yellow, indigo and orange regions of Fig. 2b. For $\bar{y}=0.8$ (white region in Fig. 2b), for most initial conditions the system converges toward states where the resident population goes extinct and only a stable level of tourists is observed: this is a full 'Disneyfication' scenario where the city turns into a tourist theme park, where the eventual level of tourists depends on initial conditions. As it could be expected, this is due to the fact that the relevance threshold for tourists is very low with respect to that for residents, and consequently, tourists take over local services and resources expelling the residents. However, for very low initial levels of tourists and high enough levels of residents, there are also trajectories where residents take over the city, letting tourists go extinct or remain present at very low levels. As the relevance threshold $\bar{y}$ grows to 1.1 (yellow region in Fig. 2 b), making access to resources more demanding for tourists, we witness the emergence of a stable attractor where residents and tourists stably coexist in the long term, approaching this state through a cyclical adjustment path, whose basin of attraction is delimited by the 


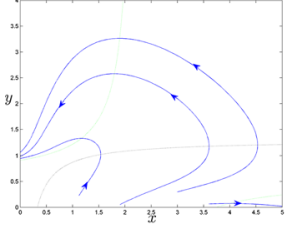

(a) $\bar{y}=.8$

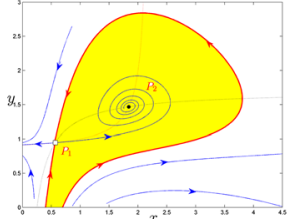

(b) $\bar{y}=1.1$

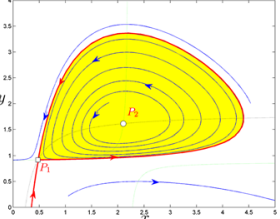

(c) $\bar{y}=1.3151$

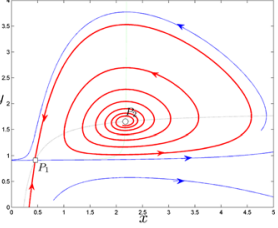

(d) $\bar{y}=1.35$

Fig. 3 Phase portrait for the system (1)-(2). Parameters are: $a=2, b=7, c=1.5, d=2.5, e=5$, $f=16.5, r=3, s=15, \bar{x}=4$

yellow region. Outside this basin, depending on the initial level of tourists vs. residents we find as before that either tourists take over entirely, or residents do, entirely or partially (that is, with a more or less high level of tourists observed in the long term). As $\bar{y}$ is brought further up at 1.3151 (indigo region), the stationary state becomes unstable and cyclical behaviors emerge within the yellow region, whereas outside the region one still observes as before, depending on initial conditions, the eventual takeover of tourists or the emergence of a state with high levels of residents and some tourists. Finally, with $\bar{y}$ at 1.35 (orange region), the system is destabilized, the stationary state is unstable and the trajectories may entail big oscillations where, despite that both the noresidents and prevailing-residents long-term states can materialize as before, it is also possible that the limit state is reached through expanding fluctuations. In particular, it is interesting to observe that as the conditions for accessibility of resources for tourists become more demanding as $\bar{y}$ increases, the resulting dynamic behaviors do not simply favor residents - rather, what we observe is an increase of the system's dynamic variability with the eventual emergence of cyclically diverging behaviors where big changes in the levels of residents vs. tourists are observed in time.

In Fig. 4 we highlight a different phenomenon, namely how the size of the basin of attraction of the stable stationary state varies with the variation of $\bar{y}$ for a given value of $\bar{x}$. We now fix $\bar{x}=2.2$ and choose the values of $\bar{y}$ in order to always remain within the yellow region of Fig. $2 \mathrm{~b}$ where a stable stationary state (attractor) exists. As we see, in Fig. 4a, as $\bar{y}$ increases, the size of the basin of attraction of the stable stationary state (denoted with a black dot) significantly increases. In Fig. 4b, we analogously set $\bar{y}$ at a constant value 1.2 and let $\bar{x}$ vary. In this case, as with the increase in $\bar{x}$ access to resources becomes less and less easy for residents, the size of the basin of attraction of the stable stationary state gradually shrinks. Maintaining a viable access to resources for residents therefore causes, as one might expect, a dynamic stabilization of the system.

Figure 5 reports yet another angle of analysis, namely, how the coordinates of the stationary state vary with $\bar{y}$ for a given level of $\bar{x}$ and of $e$. The other parameters are still kept at the usual values. We see that, as $\bar{y}$ increases, the stationary state entails smaller equilibrium levels of both tourists and residents. However, for a given $\bar{y}$, increases in $\bar{x}$ imply lower levels of tourists at the stationary state. This pattern of course only informs us about the composition of the stationary state but not about its stability properties or, if attractive, about the size of its basin of attraction. In Fig. 5b, as it could be expected, as $c$ grows, we see that the stationary state entails lower and lower 

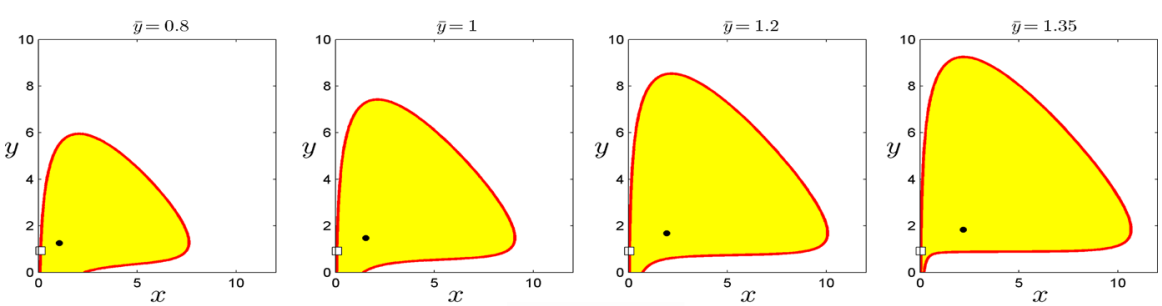

(a) $\bar{x}=2.2$
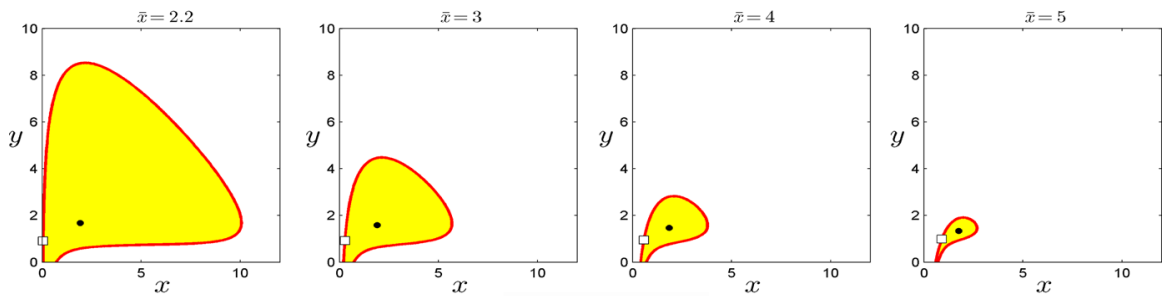

(b) $\bar{y}=1.2$

Fig. 4 Parameters are: $a=2, b=7, c=1.5, d=2.5, e=5, f=16.5, r=3, s=15$

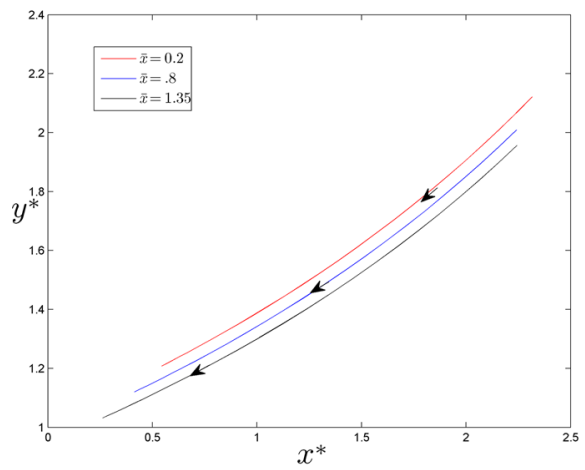

(a) varying $\bar{y}$

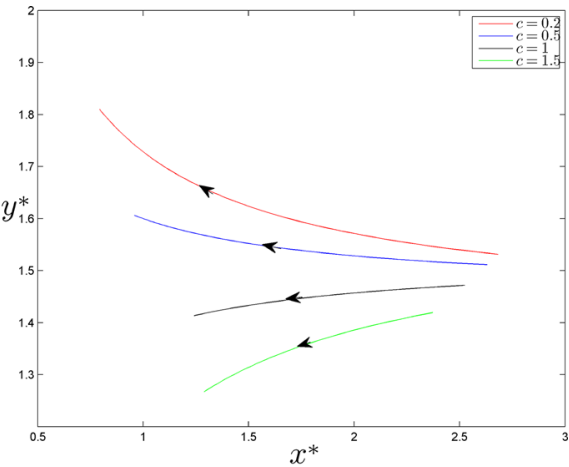

(b) varying $e$

Fig. 5 The arrows along the curves show the direction of the change in the stationary state values of $x^{*}$, $y^{*}$ as $\mathbf{a} \bar{y}, \mathbf{b} e$ increase, keeping all other parameters constant. Parameters are: $a=2, b=7, c=1.5$, $d=2.5, e=5, f=16.5, r=3, s=15$

levels of tourism all other things being equal. Beyond a certain threshold for $c$, the steady state level of tourists keeps declining even when $e$ increases, whereas below the threshold an increase in $e$ causes a corresponding increase of the level of tourists at the steady state.

We have checked the robustness of our simulation results through further, extensive numerical tests that are not reported here for brevity and which confirm our analysis. 


\section{Discussion and conclusions}

We have built a simple model to study the conditions for the emergence of overtourism through mathematical simulation of a predator-prey-inspired dynamical system. The core element that drives our dynamics is the competition for the accessibility of resources and services between residents and tourists, a feature that is typical of overtourism and is mainly responsible for its most disruptive effects. The model has been further enriched with a few elements that capture effects such as tourist congestion or the experience value for tourists deriving from the interaction with residents or from the intrinsic attractiveness of the city. Even if studying the model in its most essential form, the dynamic analysis is challenging.

Our model shows that, under suitable conditions, overtourism may emerge, to the point of causing a full 'Disneyfication' of the city with the eventual extinction of all residents and its final transformation into a tourist theme park. However, also the reverse option is possible, with tourists disappearing from the city or reaching a stable level without taking over the local economy. Of course, in addition to these extreme cases, the possibility of a stable coexistence of residents and tourists is also possible, but equally possible are more complex dynamics that may entail stable cyclical oscillations or wide variations in the relative levels of the populations of residents and tourists. The outcome that is eventually reached depends on a very complex constellation of parameters, each of which plays a specific role that can, however, be fully understood only by means of a thorough analysis.

What we have learnt from this study is that, in a nonlinear setting, acting on specific parameter values may cause counterintuitive effects. As we have seen, some cities have decided to tackle overtourism by restricting tourists' access to local services and resources. In our model, this basically amounts to raising the relevance threshold of tourism as it makes the conditions for access to tourist-specific resources more demanding. However, this does not necessarily entail the eventual reduction of the number of tourists or even the reaching of a stable stationary state where the number of tourists is under control. It may happen instead that the main effect of raising the threshold $\bar{y}$ is destabilizing the system, for instance by causing the emergence of large oscillations in the levels of residents and tourists. This means that, contrary to commonsense approaches, it is important to understand how certain measures affect the whole structural organization of the local economy. The interplay with factors such as congestion, intrinsic attractiveness, or experience value can generate complex dynamic effects that influence the existence and stability of stationary states, and more generally the dynamic behavior of the system.

It is interesting to notice that, in determining the existence and stability properties of the stationary states of the model, certain composite parameters play a more substantial role than others. In particular, residents' susceptibility $(B)$ is the key parameter in determining the dynamic regime that prevails, whereas residents' resilience $(A)$ and tourists' susceptibility $(C)$ play practically no role, although it is far from excluded that they may play a role in the dynamic behavior of the system far from equilibrium. The central point seems therefore to be the conditions for access to local services and resources by residents. Promoting residents' access does not merely amount to restricting access to the same resources to tourists. Lowering residents' susceptibility 
might be a better strategy and also a source of stabilization of the system. This goal may be reached, for instance, by providing better social and welfare services to residents, by supporting social entrepreneurship that better addresses critical local needs, by improving the quality of key resident services such as kindergartens or retirement houses, and so on. What is important to stress is that, in a nonlinear system, even relatively small changes may make a big difference, for better or for worse. And therefore, building models that allow to estimate the likely impact of policy measures as an essential support tool for public decision making becomes crucial.

It is unlikely that overtourism will be successfully dealt with by cities through the implementation of occasional measures without a clear evidence-based strategy that is informed by a solid knowledge of the underlying system of structural interdependencies, not unlike what happens in the management of ecological systems. Our study has clear limitations, due to the extreme simplicity of the model that disregards many potentially relevant factors. In particular, the role of residents' and tourists' expectations and attitudes, that as we have seen is an important aspect in the current evaluation of the social and economic impacts of tourism, could also be modeled with all the ensuing complexities arising from cultural transmission effects, misperceptions and biases, manipulation of consensus, and so on. Another important limitation is that an empirical estimation of the values of the model parameters is not simple and would call for a sophisticated nonlinear econometric analysis. Data availability is also demanding, as, ideally, very long time series of residents/tourists populations of cities with significant or potential overtourism issues would be required. The nonlinearity features of the model would imply that even relatively small estimation errors might have big consequences on the projected dynamics, yielding potentially misleading indications. The present paper has therefore mainly a conceptual value in drawing attention upon the dynamic complexity of the socioeconomic dynamics of overtourism, and the ensuing necessity to carefully assess the long-term effects of policy changes even when they intuitively seem to respond effectively to outstanding issues. Curbing tourist congestion through the reduction of commercial licenses for tourism-related businesses, for instance, looks like an appealing solution but its long-term consequences might be more complex than one could expect, depending on the overall structure of the local economy and its 'ecosystemic' interdependencies. In its current form, our model is not tailored to guiding policy design choices, a task that requires suitably calibrated empirical models. But we hope that this first study may inspire further, more sophisticated analyses that will serve in turn as a guide for the construction of policy oriented tools. We look forward to this promising perspective.

Funding Open access funding provided by Università degli Studi di Sassari within the CRUI-CARE Agreement. The research of Angelo Antoci and Paolo Russu was supported by the Italian Ministry of Education (MIUR) ("Dipartimenti di Eccellenza" Program 2018-2022), and by the University of Sassari ("Fondo di Ateneo per la ricerca 2020"). 


\section{Appendix}

\subsection{Hopf bifurcation}

The Jacobian matrix of the system (1)-(2) evaluated at a stationary state $P^{*}=\left(x^{*}, y^{*}\right)$ is given by:

$$
J\left(P^{*}\right)=\left(\begin{array}{ll}
a+b \bar{y}+c-b y^{*} & -b x^{*} \\
-\bar{y} e+(e+d) y^{*} & (e+d) x^{*}-f
\end{array}\right)
$$

Liu (1994) derived a criterion to prove the existence of a Hopf bifurcation without using the eigenvalues of the matrix $J\left(P^{*}\right)$. According to Liu's criterion, if the stationary state $P^{*}$ depends smoothly upon a parameter $p \in(0, \bar{p})$, and there exists a parameter value $p_{H} \in(0, \bar{p})$ such that the characteristic equation of $J\left(P^{*}\right), \lambda^{2}+T(p) \lambda+\Delta(p)=0$, satisfies the conditions:

1. $T(p)$ and $\Delta(p)$ are smooth functions of $p$ in an open interval containing $p_{H}$,

2. $T\left(p_{H}\right)=0, \Delta\left(p_{H}\right)>0$,

3. $\left(\frac{d T(p)}{d p}\right)_{p=p_{H}} \neq 0$,

then a Hopf bifurcation occurs at $p=p_{H}$.

Focusing on parameter $\bar{y}$, the condition 3 of Liu's criterion becomes:

$$
\frac{d T(\bar{y})}{d \bar{y}}=\left(-b-b \frac{d y^{*}(\bar{y})}{d \bar{y}}-(e+d) \frac{d x^{*}(\bar{y})}{d \bar{y}}\right)_{\bar{y}=\bar{y}_{H}} \neq 0
$$

and likewise for the other parameters of the model. It is rather cumbersome to check that condition (3) is satisfied without specifying parameter values. We therefore pose: $a=2 b=7, c=1.5, d=2.5, e=5, f=16.5, r=3, s=15, \bar{x}=3$, and consider $\bar{y}$ as the bifurcation parameter. For this set of parameter values, the coordinates of the stationary state with the highest values of $x$ and $y$ in Fig. 1 are given by:

$$
\begin{aligned}
x^{*}(\bar{y}) & =\frac{3}{35} M(\bar{y}) \\
y^{*}(\bar{y}) & =\frac{5}{3} \frac{-\frac{3}{2}+\frac{3}{10} M(\bar{y})+\frac{3}{5} M(\bar{y}) \bar{y}}{M(\bar{y})}
\end{aligned}
$$

where $M(\bar{y})=\frac{-24+77 \bar{y}+\sqrt{-579.0-4466.0 \bar{y}+5929.0 \bar{y}^{2}}}{3.0+2.0 \bar{y}}$, and $x^{*}(\bar{y}), y^{*}(\bar{y})>0$ if $\bar{y} \in$ $(0.8660115242,+\infty)$.

In such a context, the equation:

$$
T(\bar{y})=13-\frac{15}{2} x^{*}(\bar{y})-7 \bar{y}+7 y^{*}(\bar{y})=0
$$


is satisfied at the value $\bar{y}_{H}=1.309562086$. Replacing such a value in the equation:

$$
\Delta(\bar{y})=\frac{105}{4} x^{*}(\bar{y})-\frac{231}{4}+\frac{231}{2} y^{*}(\bar{y})-\frac{231}{2} \bar{y}+\frac{35}{2} \bar{y} x^{*}(\bar{y})
$$

we obtain $\Delta\left(\bar{y}_{H}\right)=91.73884257>0$, and therefore, condition 2 holds.

Finally, at $\bar{y}=\bar{y}_{H}$, we have $\frac{d T(\bar{y})}{d \bar{y}}=-12.92610372 \neq 0$, so that a Hopf bifurcation occurs at the parameter value $\bar{y}_{H}=1.309562086$.

\subsection{Existence and stability of stationary states}

In order to study the existence of the stationary states, we rewrite the system (1)-(2) as follows:

$$
\begin{aligned}
& \dot{x}=G(x, y):=b A\left(-B+x-\frac{x y}{A}\right) \\
& \dot{y}=H(x, y):=\frac{f}{E}(D-C x y+x y-E y)
\end{aligned}
$$

so, the isoclines (i.e., $g(x)=0$ and $h(x)=0$ ) of the dynamical system become:

$$
\begin{aligned}
& y=g(x):=\frac{A}{x}(x-B) \\
& y=h(x):=C \frac{x-D}{x-E} .
\end{aligned}
$$

It is easy to check that the above functions are two hyperbolas with the following properties:

i. the function $y=g(x)$ (Fig. 6a, b) presents an horizontal asymptote at $y=A$, a vertical one at $x=0$ and its graph crosses the $x$-axis at $\hat{x}=B(\operatorname{sign}(B)=$ $\operatorname{sign}(c \bar{x}-r))$;

ii. the function $y=h(x)$ (Fig. 6c, d) presents an horizontal asymptote at $y=C$, a vertical one at $x=E$ and its graph crosses the $x$-axis at $\hat{x}=D$.

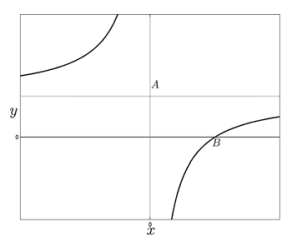

(a) $y=g(x), B>0$

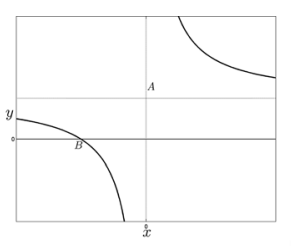

(b) $y=g(x), B<0$.

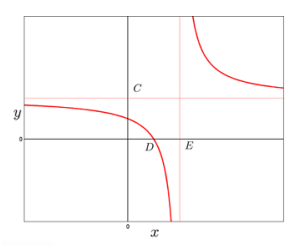

(c) $y=h(x), D>E$.

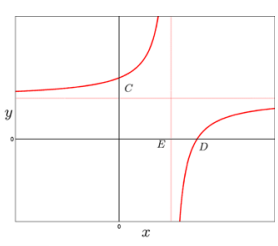

(d) $y=h(x), D>E$.

Fig. 6 Shapes of the hyperbola (nullcline) of equation: $\mathbf{a}, \mathbf{b} y=g(x), \mathbf{c}, \mathbf{d} y=h(x)$ 


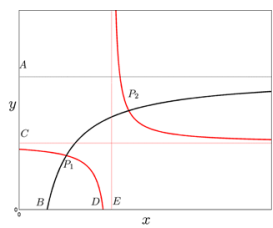

(a) $S_{1}: 0<B<D<E$

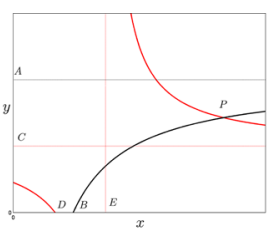

(b) $S_{2}: D<B<E$

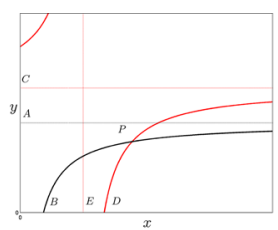

(c) $S_{3}: 0<B<E<D$

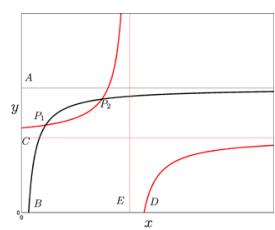

(d) $S_{4}: B<E<D$

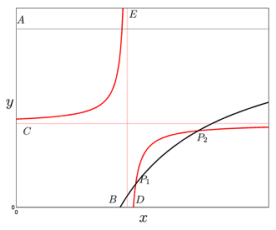

(e) $S_{5}: B<E<D$

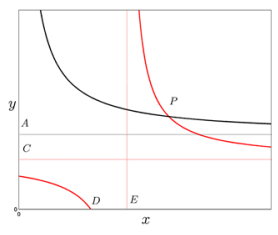

(f) $S_{6}: B<0, D<E$

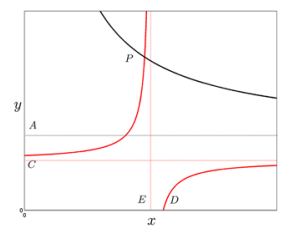

(g) $S_{7}: B<0, D>E$

Fig. 7 All possible intersection between the two nullclines, shown in Fig. 6

Remark 1 The graphs of $g(x)$ and $h(x)$ can have at most two intersection points and, therefore, at most two stationary states exist. Furthermore, under the assumption that all parameters of the system (1)-(2) are strictly positive, it is easy to check that the inequality $A>B$ is always satisfied.

Overlapping the pairs of Fig. 6a-c, a-d and b-d, we obtain all possible intersections between the two isoclines as shown in Fig. 7a-g. This proves the claim about the existence of the stationary states of Proposition 1.

In order to study the stability properties of the stationary states, we compute the Jacobian matrix of the system (4)-(5), evaluated at the stationary state $P^{*}$ :

$$
J\left(P^{*}\right)=\left(\begin{array}{ll}
b\left(A-y^{*}\right) & -b x^{*} \\
\frac{f}{E}\left(y^{*}-C\right) & \frac{f}{E}\left(x^{*}-E\right)
\end{array}\right)
$$

We know that the signs of the determinant $\mathrm{D}\left(J\left(P^{*}\right)\right)=b \frac{f}{E}$ $\left[x^{*}\left(y^{*}-C\right)-\left(y^{*}-A\right)\left(x^{*}-E\right)\right]$ and the trace $\mathrm{T}\left(J\left(P^{*}\right)\right)=\frac{f}{E}\left(x^{*}-E\right)-b\left(y^{*}-A\right)$ of the matrix (8) give us the stability properties of the stationary state. In particular, if $\mathrm{D}\left(J\left(P^{*}\right)\right)<0$, then the stationary state is a saddle point; if $\mathrm{D}\left(J\left(P^{*}\right)\right)>0$ and $\mathrm{T}\left(J\left(P^{*}\right)\right)>0(<0)$, the stationary state is a repeller (an attractor) point.

We prove the result for the sub-regime $0<B<E<D$ (see claim (1.b) in Proposition 1) shown in Fig. 7d. The other claims for the other sub-regimes can be proven in the same way.

We observe that the slopes of curves $G(x, y)=0$ and $H(x, y)=0$ are given by:

$$
m_{G}(x, y)=-\frac{y-A}{x} \quad m_{H}(x, y)=-\frac{y-C}{x-E}
$$


at any given stationary state $P^{*}$.

In this respect, we rewrite the determinant as $\mathrm{D}\left(J\left(P^{*}\right)\right)=b \frac{f}{E} x^{*}\left(x^{*}-\right.$ E) $\left(m_{G}\left(x^{*}, y^{*}\right)-m_{H}\left(x^{*}, y^{*}\right)\right)$

Since $y^{*}-A<0, y^{*}-C>0, x^{*}-E<0$, the stability analysis can be developed as follows:

i. At the stationary state $P_{1}$, the curves $G=0$ and $H=0$ are both increasing and the slope of $G=0$ is greater than that of $H=0$. Then, the determinant $\mathrm{D}\left(J\left(P_{1}\right)\right)$ is strictly negative and the stationary state is a saddle.

ii. At the stationary state $P_{2}$, the curves $G=0$ and $H=0$ are both increasing and the slope of $H=0$ is greater than that of $G=0$. Then, the determinant $\mathrm{D}\left(J\left(P_{2}\right)\right)$ is strictly positive and the stationary state is either a repeller or an attractor depending on the sign of the trace $\mathrm{T}\left(J\left(P_{2}\right)\right)$.

\section{References}

Agyeiwaah E (2019) Over-tourism and sustainable consumption of resources through sharing: the role of government. Int J Tour Cities 6:99-116

Alam MS, Paramati SR (2016) The impact of tourism on income inequality in developing economies: Does Kuznets curve hypothesis exist? Ann Tour Res 61:111-126

Alexis P (2017) Over-tourism and anti-tourism sentiment: an exploratory analysis and discussion. Ovidius Univ Ann Econ Sci Ser 17:288-293

Almeida Garcia F, Balbuena Vazquez A, Cortes Macias R (2015) Resident's attitudes toward the impacts of tourism. Tour Manag Perspect 13:33-40

Aref F, Redzuan MB (2009) Community capacity building for tourism development. J Hum Ecol 27:21-25

Benner M (2019) From overtourism to sustainability: a research agenda for qualitative tourism development in the Adriatic. Munich Personal RepEc Archive, Munich, p 92213

Boley BB, Gard McGehee N, Hammett ALT (2017) Importance-performance analysis (IPA) of sustainable tourism initiatives: the residents perspective. Tour Manag 58:66-77

Boztas S (2017) Amsterdam bans new tourist shops to combat 'Disneyfication' of the city. The Telegraph, London

Carvalho FL, Guerreiro M, Matos N (2020) Overtourism: a systematic review of literature. Handbook of research on impacts, challenges, and policy responses to overtourism. IGI Global, Hershey, pp 12-36

Caserta S, Russo AP (2002) More means worse: asymmetric information, spatial displacement and sustainable heritage tourism. J Cult Econ 26:245-260

Cheer JM, Milano C, Novelli M (2019) Tourism and community resilience in the anthropocene: accentuating temporal overtourism. J Sustain Tour 27:554-572

Chen Z, Li L, Li T (2017) The organizational evolution, systematic construction and empowerment of Langde Miao's community tourism. Tour Manag 58:276-285

Cole S (2006) Information and empowerment: the keys to achieving sustainable tourism. J Sustain Tour 14:629-644

Cole S (2007) Beyond authenticity and commodification. Ann Tour Res 34:943-960

Concu N, Atzeni G (2012) Conflicting preferences among tourists and residents. Tour Manag 33:1293-1300

Conte R (2018) Firenze, sfrattati cinquecento fiorentini per far posto ai turisti. La Nazione

Easterling DS (2004) The residents' perspective in tourism research. J Travel Tour Market 17:45-62

Economist (2018) Wish you weren't here. The backlash against overtourism. The Economist, p 27

Ganzaroli A, De Noni I, van Baalen P (2017) Vicious advice: analyzing the impact of TripAdvisor on the quality of restaurants as part of the cultural heritage of Venice. Tour Manag 61:501-510

Henley J (2019) Ten cities ask EU for help to fight Airbnb expansion. The Guardian, London

Higgins-Desbiolles F (2020) The end of global travel as we know it: an opportunity for sustainable tourism. The Conversation, March 17. https://theconversation.com/the-end-of-global-travel-as-we-know-itan-opportunity-for-sustainable-tourism- 133783 
Hinsliff G (2018) Airbnb and the so-called sharing economy is hollowing out our cities. The Guardian, London

Jang H, Park M (2020) Social media, media and urban transformation in the context of overtourism. Int J Tour Cities 6:233-260

Kuscer K, Mihalic T (2019) Residents' attitudes toward overtourism from the perspective of tourism impacts and cooperation-The case of Ljubljana. Sustainability 11:1823

Lacy JA, Douglass WA (2002) Beyond authenticity: the meaning and uses of cultural tourism. Tour Stud 2:5-21

Ledsom A (2019) Barcelona is threatening to shut out tourists. Forbes, New York

Lee S (2009) Income inequality in tourism services-dependent counties. Curr Issu Tour 12:33-45

Lefebvre H (2010) Writings on cities. Blackwell, Oxford

Lemmi E, Sacco PL, Crociata A, Agovino M (2018) The Lucca Comics and Games Festival as a platform for transformational cultural tourism: evidence from the perception of residents. Tour Manag Perspect 27:162-173

Lin Z, Chen Y, Filieri R (2017) Resident-tourist value co-creation: the role of residents' perceived tourism impacts and life satisfaction. Tour Manag 61:436-442

Liu WM (1994) Criterion of Hopf bifurcation without using eigenvalues. J Math Anal Appl 182:250-256

Marcus L (2019) Popular medieval Belgian town Bruges makes moves to restrict tourism. CNN Travel, Tokyo

Mathew PV, Sreejesh S (2017) Impact on responsible tourism on destination sustainability and quality of life of community in tourism destinations. J Hospital Tour Manag 31:83-89

Muler Gonzalez V, Coromina L, Gali N (2018) Overtourism: residents' perceptions of tourism impact as an indicator of resident social carrying capacity—case study of a Spanish heritage town. Tour Rev 73:277-296

Neuts B, Nijkamp P (2012) Tourist crowding perception and acceptabiity in cities. An applied modeling study on Bruges. Ann Tour Res 39:2133-2153

Nunkoo R, Gursoy D (2012) Residents' support for tourism. An identity perspective. Ann Tour Res 39:243268

O'Sullivan F (2017) Amsterdam doesn't want any more stores for tourists. CityLab

Paulaskaite D, Powell R, Coca-Stefaniak JA, Morrison AM (2017) Living like a local: authentic tourism experiences and the sharing economy. Int J Tour Res 19:619-628

Picascia S, Romano A, Teobaldi M (2019) The airification of cities. Making sense of the impact of peer to peer short term letting on urban functions and economy. SocArXiv

Pinkster FM, Boterman WR (2017) When the spell is broken: gentrification, urban tourism, and privileged discontent in the Amsterdam canal district. Cult Geogr 24:457-472

Prebensen NK, Xie J (2017) Efficacy of co-creation and mastering on perceived value and satisfaction in tourists' consumption. Tour Manag 60:166-176

Rasoolimanesh SM, Ringle CM, Jaafar M, Ramayah T (2017) Urban vs. rural destinations: Residents' perceptions, community participation and support for tourism development. Tour Manag 60:147-158

Ribeiro MA, Pinto P, Silva JA, Woosnam KM (2017) Residents' attitudes and the adoption of pro-tourism behaviors: the case of developing island countries. Tour Manag 61:523-537

Segota T, Mihalic T, Kuscer K (2017) The impact of residents' informedness and involvement on their perceptions of tourism impacts: the case of Bled. J Destin Market Manag 6:196-206

Seraphin H, Sheeran P, Pilato M (2018) Over-tourism and the fall of Venice as a destination. J Destin Market Manag 9:374-376

Sharpley R (2014) Host perceptions of tourism: a review of the research. Tour Manag 42:37-49

Simpson MC (2009) An integrated approach to assess the impacts of tourism on community development and sustainable livelihoods. Commun Dev J 44:186-208

Spinks R (2018) All the things tourists are not allowed to do in Venice. Quartz, New York

Stylidis D, Biran A, Sit J, Szivas EM (2014) Residents' support for tourism development: the role of residents' place image and perceived tourism impacts. Tour Manag 45:260-274

Tantucci E (2018) Venezia, raddoppiati in un solo anno alloggi turistici e bed and breakfast. La Nuova Venezia, Venice

Truong VD, Hall CM, Garry T (2014) Tourism and poverty alleviation: perceptions and experiences of poor people in Sapa, Vietnam. J Sustain Tour 22:1071-1089

van den Borg J, Costa P, Gotti G (1996) Tourism in European heritage cities. Ann Tour Res 23:306-321 
Vargas-Sanchez A, Porras-Bueno N, de los Angeles Plaza-Mejia M (2011) Explaining residents' attitudes to tourism. Is a universal model possible? Ann Tour Res 38:460-480

Wang S, Chen JS (2015) The influence of place identity on perceived tourism impacts. Ann Tour Res 52:16-28

Wiley M (2019) This Croatian city is struggling with overtourism. Is game of thrones to blame?. Business Insider, Venice

Zhu Y (2012) Performing heritage: rethinking authenticity in tourism. Ann Tour Res 39:1495-1513

Publisher's Note Springer Nature remains neutral with regard to jurisdictional claims in published maps and institutional affiliations. 